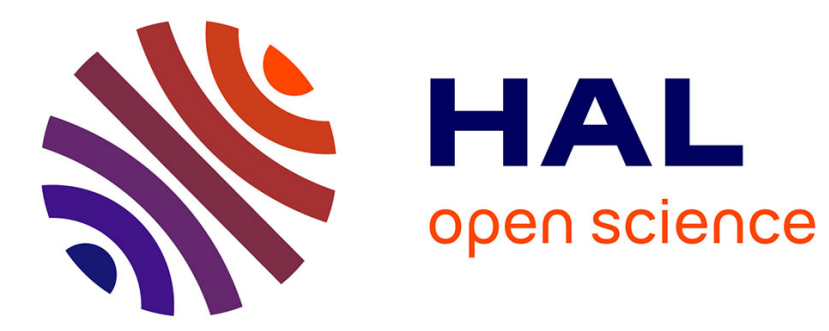

\title{
MRI Based Bayesian Personalization of a Tumor Growth Model
}

\author{
Matthieu Lê, Hervé Delingette, Jayashree Kalpathy-Cramer, Elizabeth R \\ Gerstner, Tracy Batchelor, Jan Unkelbach, Nicholas Ayache
}

\section{To cite this version:}

Matthieu Lê, Hervé Delingette, Jayashree Kalpathy-Cramer, Elizabeth R Gerstner, Tracy Batchelor, et al.. MRI Based Bayesian Personalization of a Tumor Growth Model. IEEE Transactions on Medical Imaging, 2016, 35 (10), pp.2329-2339. 10.1109/TMI.2016.2561098 . hal-01324849

\section{HAL Id: hal-01324849 \\ https://hal.inria.fr/hal-01324849}

Submitted on 1 Jun 2016

HAL is a multi-disciplinary open access archive for the deposit and dissemination of scientific research documents, whether they are published or not. The documents may come from teaching and research institutions in France or abroad, or from public or private research centers.
L'archive ouverte pluridisciplinaire HAL, est destinée au dépôt et à la diffusion de documents scientifiques de niveau recherche, publiés ou non, émanant des établissements d'enseignement et de recherche français ou étrangers, des laboratoires publics ou privés. 


\title{
MRI Based Bayesian Personalization of a Tumor Growth Model
}

\author{
Matthieu Lề ${ }^{1}$, Hervé Delingette ${ }^{1}$, Jayashree Kalpathy-Cramer ${ }^{2}$, Elizabeth R. Gerstner ${ }^{3}$, \\ Tracy Batchelor ${ }^{3}$, Jan Unkelbach ${ }^{4}$, Nicholas Ayache ${ }^{1}$
}

\begin{abstract}
The mathematical modeling of brain tumor growth has been the topic of numerous research studies. Most of this work focuses on the reaction-diffusion model, which suggests that the diffusion coefficient and the proliferation rate can be related to clinically relevant information. However, estimating the parameters of the reaction-diffusion model is difficult because of the lack of identifiability of the parameters, the uncertainty in the tumor segmentations, and the model approximation, which cannot perfectly capture the complex dynamics of the tumor evolution. Our approach aims at analyzing the uncertainty in the patient specific parameters of a tumor growth model, by sampling from the posterior probability of the parameters knowing the magnetic resonance images of a given patient. The estimation of the posterior probability is based on: i) a highly parallelized implementation of the reaction-diffusion equation using the Lattice Boltzmann Method (LBM), and ii) a high acceptance rate Monte Carlo technique called Gaussian Process Hamiltonian Monte Carlo (GPHMC). We compare this personalization approach with two commonly used methods based on the spherical asymptotic analysis of the reaction-diffusion model, and on a derivativefree optimization algorithm. We demonstrate the performance of the method on synthetic data, and on seven patients with a glioblastoma, the most aggressive primary brain tumor. This Bayesian personalization produces more informative results. In particular, it provides samples from the regions of interest and highlights the presence of several modes for some patients. In contrast, previous approaches based on optimization strategies fail to reveal the presence of different modes, and correlation between parameters.
\end{abstract}

Index Terms-tumor growth, glioblastoma, reaction-diffusion, personalization, bayesian, LBM, Monte Carlo

\section{INTRODUCTION}

\section{A. Motivations}

$\mathbf{T}$ HE objectives of the mathematical modeling of brain tumor growth are fourfold. First, it could help to better understand the mechanisms behind the disease progression [1]. Second, the personalization of such models to specific patients could allow one to quantify the aggressiveness of the tumor, which has been shown to be correlated with clinically relevant information [2], [3]. Third, personalized models could help predicting the future evolution of a given tumor [4]. Fourth, personalized models could lead the way toward objective and more efficient personalized therapy. For instance, it has already been used in order to personalized radiotherapy plans [5], [6].

\footnotetext{
${ }^{1}$ Asclepios Project, Inria Sophia Antipolis, France.

${ }^{2}$ Martinos Center for Biomedical Imaging, Harvard-MIT Division of Health Sciences and Technology, Charlestown, MA, USA.

${ }^{3}$ Department of Neurology, Massachusetts General Hospital, Boston, MA, USA.

${ }^{4}$ Department of Radiation Oncology, Massachusetts General Hospital and Harvard Medical School, Boston, MA, USA.
}

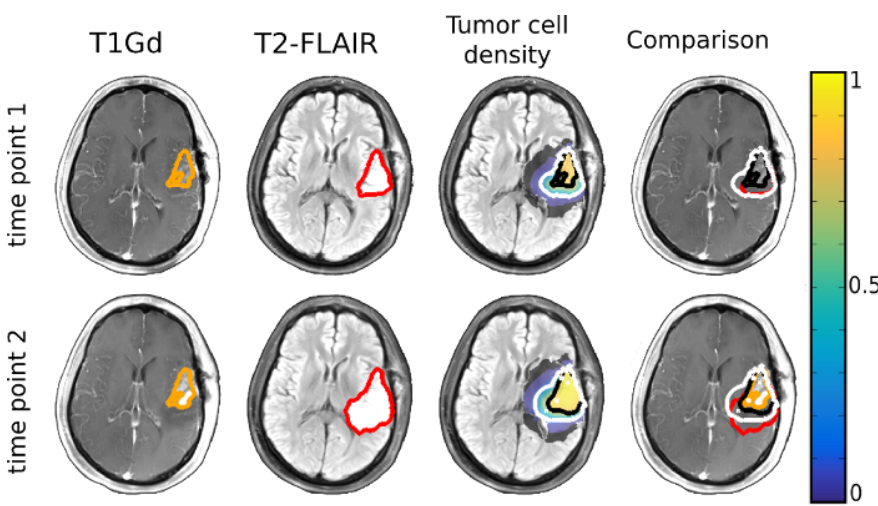

Figure 1: (Left) The proliferative rim is outlined in orange on the T1Gd MRI at two different time points; (Middle Left) The edema is outlined in red on the T2-FLAIR MRI at two different time points. The edema encloses the proliferative rim; (Middle Right) Tumor cell density computed with the reaction-diffusion model. The black (resp. white) line is the threshold values $\tau_{1}$ (resp. $\tau_{2}$ ) corresponding to the T1Gd (resp. T2-FLAIR) abnormality; (Right) Comparison between the clinician segmentation and the contours from the model.

In this article, we present a method to study the uncertainty in the patient specific parameters of a tumor growth model. To do so, we estimate the posterior probability of the parameters, which allows us to analyze the correlation between the personalized parameters of the model. Moreover, it gives valuable information on the confidence one has in the estimation and the ability of the model to explain the data. The method is based on the Bayesian personalization of a tumor growth model. We specifically apply it to the personalization of glioblastoma growth using a reaction-diffusion model. We detail the results on one synthetic - but realistic - case, and 7 patients.

\section{B. Clinical Background}

Gliomas account for $30 \%$ of primary brain and central nervous system tumors. They are characterized by their infiltrative nature: malignant cells invade the tissue by progressing along the white matter fiber tracts. They can either be low grade or high grade, and very well localized or extremely diffused. We focus on grade IV gliomas called glioblastomas multiformes (GBM). They are the most common glioma - at least 50\% of the recorded cases, and an incidence of 3.19 per 100,000 [7]. They grow extremely rapidly, and are the most malignant primary brain tumors. 


\section{Modeling: Previous Work}

The modeling of the tumor evolution can be done at different scales [8]. On the microscopic scale, the model describes the evolution of individual cells based on division and invasion rules. On the macroscopic scale, the model describes the spatio-temporal evolution of the tumor cell density in the considered domain, based on partial differential equations. Over the last 20 years, particular attention has been given to the reaction-diffusion equation,

$$
\begin{gathered}
\frac{\partial u}{\partial t}=\underbrace{\nabla(D \cdot \nabla u)}_{\text {Diffusion }}+\underbrace{\rho u(1-u)}_{\text {Logistic Proliferation }} \\
D \nabla u \cdot \vec{n}_{\partial \Omega}=0 \\
\boldsymbol{\theta}=(D, \rho)
\end{gathered}
$$

Equation (1) describes the spatio-temporal evolution of the tumor cell density $u$, which infiltrates neighboring tissues with a diffusion tensor $D$, and proliferates with a net proliferation rate $\rho$. Equation (2) enforces Neumann boundary conditions on the brain domain $\Omega$, and $\boldsymbol{\theta}$ denotes the parameters of interest of the model.

In 1989, Murray et al. [9] pioneered on the use of mathematics applied to brain tumor growth, using the reaction-diffusion equation with an exponential growth. Assuming an isotropic diffusion in an infinite domain, he relates the velocity $v$ of the tumor growth to the proliferation rate $\rho$ and the diffusion coefficient $D, v=2 \sqrt{\rho D}$. This work has lead the way to numerous other studies [10], [11], [12].

Improving the computational efficiency of tumor growth models has been the topic of several studies. In 2010, Konukoglu et al. [13] introduced an approximation of the asymptotic solution of the reaction-diffusion equation based on an Eikonal equation, which can be solved with a Fast Marching algorithm, resulting in drastically reduced computation times. In 2012, Mosayebi et al. [14] introduced a similar method to compute the tumor invasion in the brain parenchyma based on a geodesic distance computed from DTI information.

The mass effect applied by the tumor to the surrounding tissues has been modeled in 2005 by Clatz et al. [15]. They coupled the reaction-diffusion equation with a mechanical model, to take into account the mass effect, i.e. the displacement of adjacent brain tissue due to the growing tumor. They relate the diffusion tensor $D$ to the Diffusion Tensor Images (DTI), thus forcing the tumor cells to preferably follow the white matter fiber tracts. In 2008, Hogea et al. [16] proposed another approach to include mass effect, using a reactiondiffusion-advection model coupled with a Eulerian mechanical framework.

Finally, research efforts recently targeted the development of more complex models. In 2011, Swanson et al. [17] developed a model dividing the tumor cells into sub-categories: the proliferative, the invasive and the hypoxic cells. This type of complex models has been further developed by Saut et al. [18] in 2014, in which they developed a coupled PDE-based model to simulate the growth of high grade gliomas seeded in real images. Finally, the models were extended to take into account the apparition of edema during the growth [19], [20].

\section{Personalization: Previous Work}

It is of particular interest to solve the inverse problem of estimating the model's parameter $\boldsymbol{\theta}=(D, \rho)$, which can describe a specific dynamic observed for a patient. As such, the modeling effort goes hand in hand with the personalization work. For personalization, most methods rely on comparing the model estimation with the abnormalities visible on Magnetic Resonance Images (MRIs) at two different time points.

A common approach to personalize reaction-diffusion equations is based on the observation that they admit wave-like solutions whose parameters can be related to the volume of the abnormalities observed on MRIs. In 2007, Harpold et al. [21] described the personalization of a glioma growth model using the reaction-diffusion equation with an exponential growth.

Another popular approach relies on solving the inverse problem using an optimization strategy. Hogea et al. [16] formulated a PDE-constrained optimization problem to estimate the parameters of the model based on image registration. Konukoglu et al. [13] used the derivative-free optimization algorithm BOBYQA (for bounded optimization by quadratic approximation) [22] to minimize the distance between the tumor segmentations observed on the MRI, and the output of the model. In 2012, Gooya et al. [23] proposed a method for the segmentation and registration of MRIs presenting glioblastoma based on the personalization of a reaction-diffusion-advection model using a single time point acquisition.

Finally, probabilistic approaches recently gained interest to solve complex inverse problems. Menze et al. [24] was among the first to propose a Bayesian formulation for brain tumor growth personalization, based on the approximation of the posterior using sparse grids. It is interesting to note that Bayesian formulations have been used for a few years in the field of cardiac modeling [25], [26]. However, the methods usually rely on approximations of the forward model using reduced order model, such as the polynomial chaos, to make the estimation of the posterior using Markov Chain Monte Carlo (MCMC) computationally tractable.

\section{E. Contributions of the Paper}

In this paper, we propose a Bayesian method for the personalization of a tumor growth model based on the reactiondiffusion equation. We propose the use of the Lattice Boltzmann Method (LBM) to implement the tumor growth model which results in reduced computation times. This is combined with a high acceptance rate Monte Carlo technique called the Gaussian Process Hamiltonian Monte Carlo (GPHMC). Contrary to previous approaches, our method does not rely on approximations of the forward model (resp. posterior probability) using reduced order models [25], [26] (resp. sparse grid methods [24]). We compare our approach to two methods adapted from the literature. The former is based on the spherical asymptotic analysis of the forward model, inspired by the work of Swanson et al. [21], [5]. The latter is based on the gradient-free optimization method BOBYQA, and is used in the work of Konukoglu et al. [13].

This paper extends [27] with a comparison with a spherical asymptotic analysis of the personalization, and more com- 
prehensive analysis on 3 additional patients. Also, a new likelihood model based on the 95th percentile of the Hausdorff distance is used, as well as a new log-uniform prior for the parameters of interest. Finally, the parameters of the GPHMC have been updated to increase the robustness of the personalization: i) the initialization of the Gaussian process is now done with a coarse grid to ensure that the whole space of parameters is covered, ii) the parameters of the Gaussian process are set by maximizing the likelihood, iii) the noise level of the likelihood model has been reduced from $\sigma=10 \mathrm{~mm}$ in [27] to $\sigma=5 \mathrm{~mm}$ in order to increase the focus on the region of interest (i.e. the posterior is more peaked when the noise level is lower).

\section{MODEL}

\section{A. The Reaction-Diffusion Model}

Because of the logistic reaction term, equation (1) does not have a closed form solution. However, in an infinite domain, with constant proliferation rate and diffusion coefficient, equation (1) admits solutions which asymptotically behave like traveling waves with speed $v=2 \sqrt{\rho D}$ [13]. This solution has the asymptotic form $u(x, t)=u(x-v t)=u(\zeta)$. Plugging it into equation (1), we get the ordinary differential equation

$$
\mathbf{n}^{\prime} D \mathbf{n} \frac{d^{2} u}{d \zeta^{2}}+v \frac{d u}{d \zeta}+\rho u(1-u)=0
$$

where $\mathbf{n}$ is the direction of motion in the infinite domain. Moreover, linearizing equation (4) for small $u<<1$, meaning that $u(1-u) \sim u$, we get the second order linear equation

$$
\mathbf{n}^{\prime} D \mathbf{n} \frac{d^{2} u}{d \zeta^{2}}+v \frac{d u}{d \zeta}+\rho u=0
$$

which admits solutions of the form $u(\zeta)=(A \zeta+$ $B) \exp (-x / \lambda)$ with $A, B$ two constants, and $\lambda=\sqrt{D / \rho}$. This equation shows that the parameter $\lambda$ is of particular importance: it is related to the spatial decay of the tumor cell density, and it is referred to as the invisibility index [5].

In the case of GBM growth modeling, it is clinically admitted that tumor cells have higher motility in white matter, compared to gray matter. Some work has been conducted to relate the diffusion tensor $D$ to DTI - see [28], [29] for a detailed discussion. In this work, for simplification, we follow [21], and define the diffusion tensor as $D=d_{w} \mathbb{I}$ in the white matter, and $D=d_{w} / 10 \mathbb{I}$ in the gray matter, where $\mathbb{I}$ is the $3 \times 3$ identity matrix, and $d_{w}$ a scalar parametrizing the diffusion tensor. We further identify the parameter $D$ with $d_{w}$. As such, the diffusion is heterogeneous and locally isotropic. This model reproduces the infiltrative nature of the GBM, takes into account anatomical barriers (ventricles, sulci, falx cerebri), and the tumor's preferential progression along white matter tracts such as the corpus callosum.

\section{B. Model and MRIs}

Data driven GBM growth modeling is based on the acquisition of different MRI modalities routinely acquired: T1 with Gadolinium contrast agent (T1Gd) and T2 Fluid-Attenuated
Inversion Recovery (T2-FLAIR). They reveal different parts of the tumor: the active part, or proliferative rim can be observed on the $\mathrm{T} 1 \mathrm{Gd}$, while the T2-FLAIR reveals the disruption of the extacellular matrix, or edema. The T1Gd abnormality is usually included inside the larger T2-FLAIR abnormality (Figure 1). The personalization of the growth of the tumor is based on two consecutive time points, resulting in a total of four abnormalities segmented by the clinician.

We can immediately see one of the pitfalls of most models: the tumor cell density is not directly visible on T1Gd and T2-FLAIR MRIs. They rather reveal the impact of the tumor growth on the brain. In order to relate the tumor cell density $u$ - solutions of (1) - to the MRIs, the frontier of the visible abnormalities is assumed to correspond to a threshold value of the tumor cell density $u$. We note $\tau_{1}$ the value of the tumor cell density $u$ corresponding to the frontier of the T1Gd abnormality, and $\tau_{2}$ the value corresponding to the frontier of the T2-FLAIR abnormality. The invisibility index defined in the previous section is related to the distance between the boundaries of the T1Gd abnormality and the T2-FLAIR abnormality: the larger the distance, the larger the invisibility index is.

\section{Simulation}

\section{A. Preprocessing of the data}

The pre-processing of the MRIs is of particular importance (Figure 2). The T1Gd and T2-FLAIR abnormalities were segmented by a clinician, and the fractional anisotropy (FA) MRI was extracted from the DTI. First, the segmentations of the second time point $t_{2}$ are mapped on the space of the first time point $t_{1}$ as follows. i) For each visit, the T2-FLAIR and FA MRIs are rigidly registered to the T1Gd MRI [30]. ii) The T1Gd MRI at $t_{2}$ is non-linearly registered to the T1Gd MRI at $t_{1}$ using the FNIRT function of the FSL software [31], [32]. The segmentation of the tumor at $t_{2}$ is used to exclude the tumor from the similarity criterion (sum-of-squared difference) during the registration. As such, the intensity values in the tumor are ignored, and the tumor is warped in accordance with the surrounding (non-masked) tissues. iii) The resulting transformations are applied to transport the T2-FLAIR abnormalities at $t_{1}$ and $t_{2}$, and the T1Gd abnormality at $t_{2}$, on the T1Gd MRI at $t_{1}$. Second, white and gray matter, and cerebrospinal fluid (CSF) are extracted from MRIs at $t_{1}$. The pipeline is the following i) Extract the brain from the skull [33]. ii) Correct for the bias and segment the white matter, gray matter, and CSF [34]. iii) Separate the left and right hemispheres [35], iv) The voxels at the boundary between the two hemispheres are tagged as CSF, in order to prevent the tumor from invading the contra-lateral hemisphere through the falx cerebri. The voxels with high FA value $(>0.45)$ and which were tagged as white matter are not affected by this process in order to ensure that the corpus callosum stays segmented as white matter. The red circle on Figure 2 emphasizes the importance of the hemisphere separation to label the falx cerebri as CSF, and the corpus callosum as white matter. 


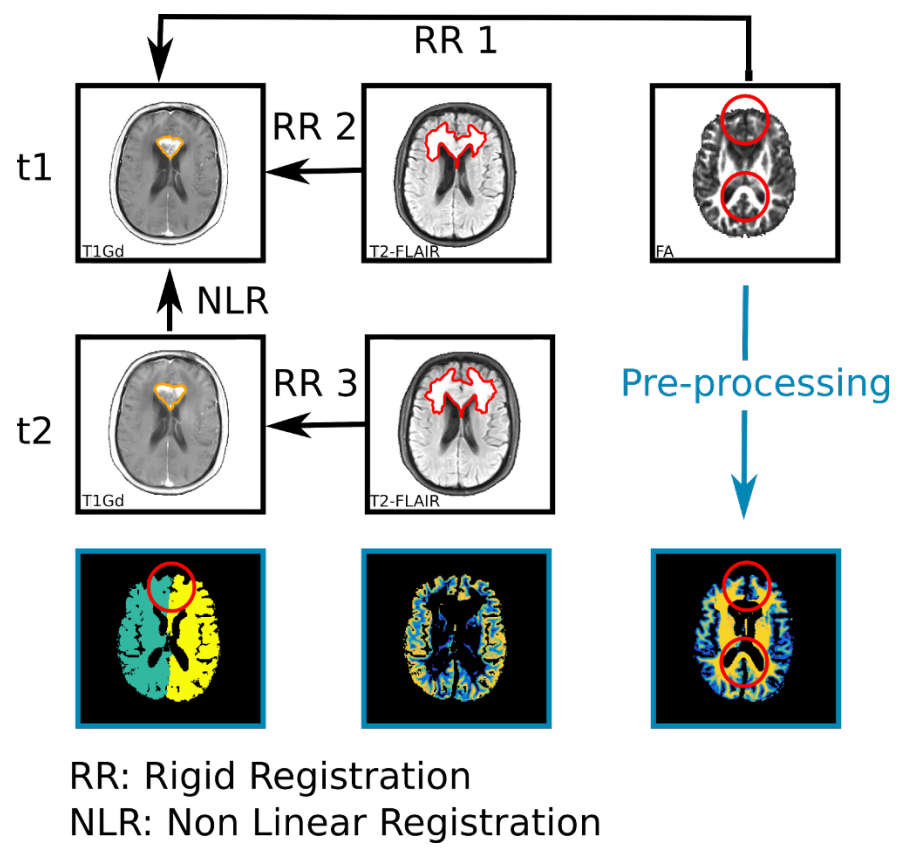

Figure 2: First row: Registration pipeline at time $t_{1}$ of the T1Gd, T2FLAIR, and FA. Middle row: Registration pipeline at time $t_{2}$ of the T1Gd, and T2-FLAIR. Bottom row, from left to right: segmentation of the left and right hemispheres to ensure the falx cerebri is labeled as CSF; gray matter segmentation; white matter segmentation.

\section{B. Lattice Boltzmann Method}

A typical approach to implement the reaction-diffusion equation is to discretize the equation using the Crank-Nicolson scheme [36]. This requires the inversion of a large sparse matrix $n \times n$ where $n$ is the number of voxels in the image, using a preconditioned gradient method like the biconjugate gradient stabilized method. For 3D MRIs with $n \sim 10^{6}$, this approach is computationally expensive. In this paper, we use the more recent explicit method called the Lattice Boltzmann Method (LBM). LBM has been successfully applied to implement the reaction-diffusion equation in the fields of cardiac electrophysiology modeling [37], [26], and liver tumor radiofrequency-ablation [38]. The idea is to model the reaction-diffusion equation as a set of fictitious particles which collide and stream on the cartesian grid. Using the D3Q7 scheme ( 3 dimensions and 7 velocities), the solution of the reaction-diffusion equation (1) is discretized $u=\sum_{\alpha=0}^{6} u_{\alpha}$, where $\alpha$ indexes the 7 different velocity directions $\left\{e_{\alpha}\right\}_{\alpha=0}^{6}$ in the 3D cartesian grid (i.e. the considered voxel and its six immediate neighbors).

$\left[e_{0}, e_{1}, e_{2}, e_{3}, e_{4}, e_{5}, e_{6},\right]=\left[\begin{array}{ccccccc}0 & 1 & -1 & 0 & 0 & 0 & 0 \\ 0 & 0 & 0 & 1 & -1 & 0 & 0 \\ 0 & 0 & 0 & 0 & 0 & 1 & -1\end{array}\right]$

Furthermore, according to the connectivity of the grid, a weight vector $\left\{w_{\alpha}\right\}_{\alpha=0}^{6}=[1 / 4,1 / 8,1 / 8,1 / 8,1 / 8,1 / 8,1 / 8]$ is defined . The algorithm, as described in [39], consists of 4 steps: i) Initialization, ii) Collision, iii) Streaming, and iv) Neumann boundary conditions. During the initialization, we set $u_{\alpha}=w_{\alpha} u$, for every $\alpha$. The collisions of the fictitious particles is then modeled with

$$
\forall \alpha \quad u_{\alpha}^{*}=u_{\alpha}-\sum_{i=0}^{6} A_{\alpha, i} u_{i}+\Delta t w_{\alpha} \rho u(1-u)
$$

where $A=M^{-1} S M \in \mathbb{R}^{7 \times 7}$ is the collision matrix. $M$ projects a vector on the moment space, and $S$ is the relaxation time matrix,

$$
\begin{aligned}
M & =\left(\begin{array}{ccccccc}
1 & 1 & 1 & 1 & 1 & 1 & 1 \\
0 & 1 & -1 & 0 & 0 & 0 & 0 \\
0 & 0 & 0 & 1 & -1 & 0 & 0 \\
0 & 0 & 0 & 0 & 0 & 1 & -1 \\
6 & -1 & -1 & -1 & -1 & -1 & -1 \\
0 & 2 & 2 & -1 & -1 & -1 & -1 \\
0 & 0 & 0 & 1 & 1 & -1 & -1
\end{array}\right) \\
S^{-1} & =\left(\begin{array}{ccccccc}
\tau_{0} & 0 & 0 & 0 & 0 & 0 & 0 \\
0 & \tau_{x x} & \tau_{x y} & \tau_{x z} & 0 & 0 & 0 \\
0 & \tau_{x y} & \tau_{y y} & \tau_{y z} & 0 & 0 & 0 \\
0 & \tau_{x z} & \tau_{y z} & \tau_{z z} & 0 & 0 & 0 \\
0 & 0 & 0 & 0 & \tau_{4} & 0 & 0 \\
0 & 0 & 0 & 0 & 0 & \tau_{5} & 0 \\
0 & 0 & 0 & 0 & 0 & 0 & \tau_{6}
\end{array}\right)
\end{aligned}
$$

with $\tau_{i j}=1 / 2 \delta_{i j}+4 \Delta t /\left(\Delta x^{2}\right) D_{i j}, \tau_{k}=1.33$, and $D$ is the diffusion tensor. The value of $\tau_{k}$ does not impact the convergence of the solution but rather the stability of the method. A value of 1.33 has been found to lead to reasonable results [37]. During the streaming step, for every $\alpha$, we have $u_{\alpha}\left(x+\delta x e_{\alpha}, t+\delta t\right)=u_{\alpha}^{*}(x, t)$. Finally, we enforce the Neumann boundary conditions with the equations

$$
\begin{aligned}
& u_{\tilde{\alpha}}(x, t+\delta t)=\frac{1}{2 \Delta} u_{\alpha}(x, t)+\frac{2 \Delta-1}{2 \Delta} u_{\tilde{\alpha}}(x, t) \\
& u_{\tilde{\alpha}}(x, t+\delta t)=2 \Delta u_{\alpha}(x, t)+(1-2 \Delta) u_{\alpha}\left(x-\Delta x e_{\alpha}, t\right)
\end{aligned}
$$

where $\tilde{\alpha}$ refers to the velocity of opposite directions than $\alpha$, and $\Delta$ is the distance between the fictitious particle and the boundary [40] (see Figure 3). We use equation (6) if $\Delta>1 / 2$ and equation (7) otherwise.

With a diffusion coefficient constant in time, the LBM does not require costly inversion of matrices. The LBM is easily parallelized such that simulating 30 days of growth, with $\delta t=$ 0.1 day, takes approximately 50 seconds on a $2.3 \mathrm{Ghz} 50$ core machine for a $1 \mathrm{~mm}$ isotropic $155 \times 182 \times 157$ grid.

\section{Initialization}

The initialization of the tumor cell density $u\left(t=t_{1}, x\right)$ at the time of the first acquisition is of particular importance, as it impacts the rest of the simulation. In this work, the tumor tail extrapolation algorithm described in [41] is used. The tumor cell density is computed outward (and inward) of the T1Gd abnormality borders as a static approximation of the wave-like solution of equation (1) with parameter $\boldsymbol{\theta}$. The algorithm is based on the recursive approximation of equation (4) for current values of the solution $u$. It only depends on the invisibility index $\lambda=\sqrt{D / \rho}$. The details of the implementation can be found in [41]. By construction 


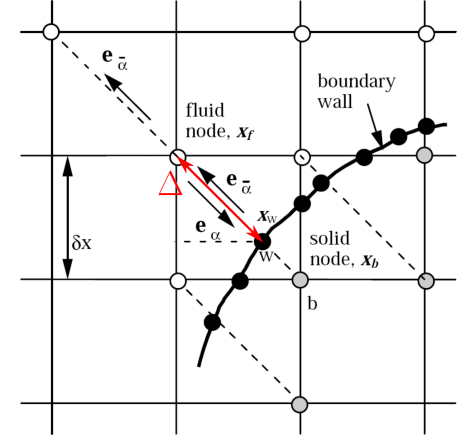

Figure 3: Cartesian grid with a boundary. Picture adapted from [40]

of the initialization, the T1Gd abnormality falls exactly on the threshold $\tau_{1}$ of the tumor cell density at the first time point (Figure 1).

\section{PERsonalization}

We describe in this section the personalization of the parameter $\boldsymbol{\theta}=(D, \rho)$ of the reaction-diffusion equation (1). Three different methods with increasing levels of complexity are presented. The first two are adapted from the literature, and are compared to the last one which we propose for the Bayesian personalization.

The first method (Section IV-A) is based on Swanson's approach [21], [5]. The spherical asymptotic analysis of the solution in a large domain is used to relate the invisibility index to the measured radii of the T1Gd and T2-FLAIR abnormalities, using multiple runs of the forward model.

The second method (Section IV-B) is based on [13] where the derivative-free optimization method BOBYQA is used to infer the parameters $D$ and $\rho$. In this article, we apply it to the case of glioblastoma where two abnormalities are observable at each time point. We do not optimize for the source point of the tumor since we initialize the model with the contour of the first T1Gd abnormality.

Finally, we propose to estimate the posterior probability of the parameters $D$ and $\rho$, knowing the clinician segmentations, using a MCMC method, the Gaussian Process Hamiltonian Monte Carlo.

\section{A. Spherical Asymptotic Analysis}

The parameters $D$ and $\rho$ can be related to the asymptotic velocity $v=2 \sqrt{D \rho}$, and the invisibility index $\lambda=\sqrt{D / \rho}$. The knowledge of the velocity and invisibility index uniquely identifies $D$ and $\rho$ since $D=v \lambda / 2$ and $\rho=v /(2 \lambda)$. As such, given a patient and the segmented T1Gd and T2-FLAIR abnormalities, we want to infer the parameters $(v, \lambda)$ from the measured radius of the abnormality through their measured volume. We hypothesize that there is a simple relationship between those radii, and the velocity and the invisibility index [21]. We consider the special case of an isotropic and homogeneous domain with a Gaussian initialization. The speed of growth can be measured as the temporal variation of the radial expansion of the abnormalities over time (Figure 4 left). Since the distinct speed of growth of the T1Gd and T2FLAIR abnormalities can be measured (and can be different), we use the geometric mean between the two, $v=\sqrt{v_{1} v_{2}}$, as an estimate. We propose in this section to find a simple relationship between the invisibility index, and the measured radii of the T1Gd and T2-FLAIR abnormalities (see Figure 4).

The tumor growth model was run on a large $201 \times 201 \times 201$ $1 \mathrm{~mm}$ isotropic grid, with different parameters $D$ and $\rho$, for 200 days, and initialized with a symmetric Gaussian. Using 15 equally spaced values of $D \in[0.021 .5] \mathrm{mm}^{2}$.day ${ }^{-1}$, and 15 equally spaced values of $\rho \in\left[\begin{array}{ll}0.002 & 0.2\end{array}\right]$ day $^{-1}, 225$ simulations were performed, keeping 11 time points per simulations. For each time point, we considered the value of the radii of the T1Gd and T2-FLAIR abnormality (resp. $r_{\mathrm{T} 2 \text {-FLAIR }}$ and $\left.r_{\mathrm{T} 1 \mathrm{Gd}}\right)$, using thresholds of $\tau_{1}=80 \%$ and $\tau_{2}=16 \%$. We observe a good linear relationship between the invisibility index $\lambda$, and the radius difference $\left(r_{\mathrm{T} 2-\mathrm{FLAIR}}-r_{\mathrm{T} 1 \mathrm{Gd}}\right)$ (Figure 5),

$$
\lambda=\sqrt{\frac{D}{\rho}}=a\left(r_{\mathrm{T} 2-\mathrm{FLAIR}}-r_{\mathrm{T} 1 \mathrm{Gd}}\right)+b
$$

with $a=0.13$ and $b=0.23 \mathrm{~mm}$ for $D$ in $\mathrm{mm}^{2} \cdot$ day $^{-1}$ and $\rho$ in day $^{-1}$. We further checked that the measured velocity corresponded to the asymptotic one (Figure 5).

In this paper, we consider the personalization using two time points. Since the invisibility index can be measured on both time points, similarly to the speed, we use the geometric mean between the two as an estimate. This personalization is based on volumetric consideration, and valid in large homogeneous domain, much like Swanson's approach [21], [5].

\section{B. BOBYQA Optimization}

The previous method does not take into account the inhomogeneity and anisotropy of the growth, and does not account for anatomical barriers such as CSF. In order to derive a finer estimation, we use an optimization method. We need to define the error corresponding to a simulation with associated parameter $\boldsymbol{\theta}$. The simulation is initialized at $t_{1}$ using the first $\mathrm{T} 1 \mathrm{Gd}$ abnormality, and simulated with the LBM until the second time point $t_{2}$ is reached. We extract the simulated contours corresponding to the thresholds $\tau_{1}$ and $\tau_{2}$, at $t_{1}$ and $t_{2}$. The 95th percentile of the symmetric Hausdorff distance between the borders of the clinician segmentation and the extracted contours is computed for: i) the T2-FLAIR abnormality at time $t_{1}$, ii) the T2-FLAIR abnormality at time $t_{2}$, iii) the T1Gd abnormality at time $t_{2}$. The mean of these distances $H_{\text {mean }}$ is used as an error measure for the simulation. We use this error measure because the Hausdorff distance is sensitive and independent to the size of the abnormality. As such, the T1Gd and T2-FLAIR will be penalized in a similar fashion. Other error measures could be considered such as the DICE coefficient, which is however sensitive to the size of the abnormalities. We minimize the error using the derivative-free optimization algorithm BOBYQA [22], using the implementation of the library NLopt ${ }^{1}$. The algorithm is

\footnotetext{
${ }^{1}$ Steven G. Johnson, The NLopt nonlinear-optimization package, http://abinitio.mit.edu/nlopt
} 


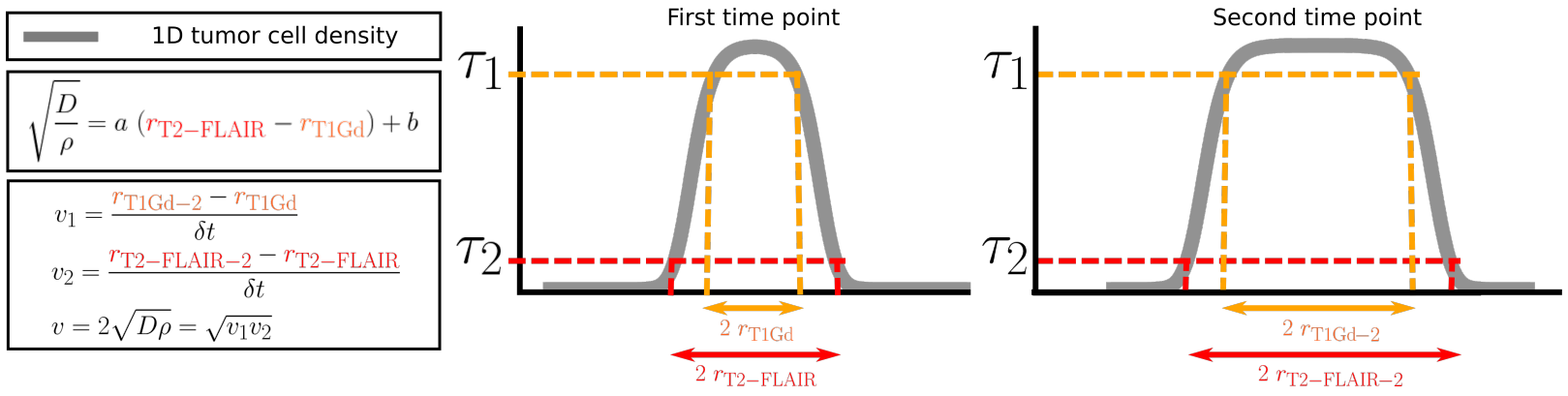

Figure 4: 1D graphical explanation detailing how the measured radii are related to the invisibility index and speed of growth of the tumor. (Left) Relationship between the invisibility index and the speed of the tumor, and the parameters $D$ and $\rho$ of the model. (Right) 1D tumor cell density at two different time points detailing how the measured radii are related to the parameters of the model.
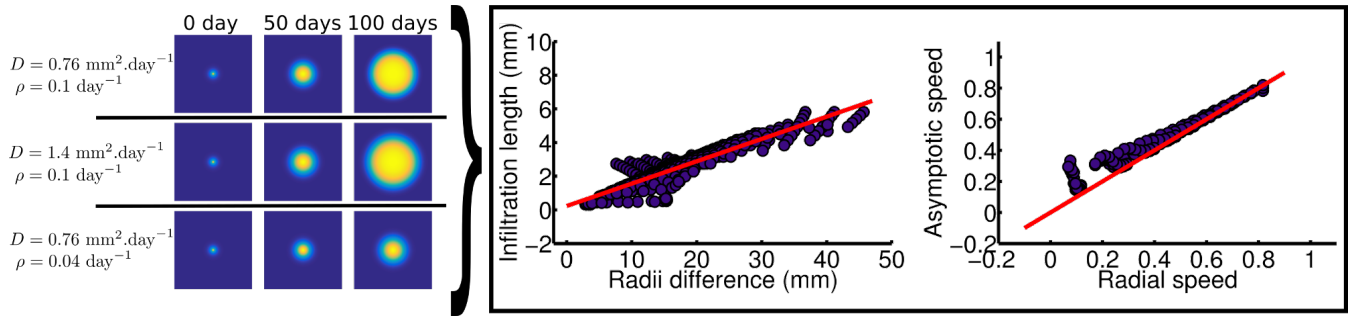

Figure 5: (Left) Example of simulations on a large cube with different parameters $D$ and $\rho$ used to infer the relationship between the invisibility index and the measured radii. (Middle) Invisibility index $(\sqrt{D / \rho})$ function of $r_{\mathrm{T} 2-\mathrm{FLAIR}}-r_{\mathrm{T} 1 \mathrm{Gd}}$. The blue dots result from the LBM simulations in the square, the red line is the linear fit. (Right) Asymptotic speed $(2 \sqrt{D \rho})$ function of the measured radial growth speed. The blue dots result from the LBM simulations, the red line is the first bisector.

run 9 times with 9 different initializations to explore various local minima. We keep the best of the 9 solutions. This personalization is in the spirit of Konukoglu's work [13], but applied to glioblastomas.

\section{Bayesian Personalization}

We denote by $S$ the set of clinician segmentations the model should fit. We are interested in the posterior probability of the model parameter $\boldsymbol{\theta}=(D, \rho)$, knowing the observations $S$. To cast the problem in a probabilistic framework, we follow Bayes rule: $P(\boldsymbol{\theta} \mid S) \propto P(S \mid \boldsymbol{\theta}) P(\boldsymbol{\theta})$. The likelihood is modeled as $P(S \mid \boldsymbol{\theta}) \propto \exp \left(-H_{\text {mean }}^{2} / \sigma^{2}\right)$, where the distance $H_{\text {mean }}$ is the mean of the 95th percentile symmetric Hausdorff distance between the border of the segmentations $S$ and the isolines of the simulated tumor cell density $u$ using $\boldsymbol{\theta}$, and the thresholds $\tau_{1}$ and $\tau_{2}$. As such, the negative $\log$-likelihood $H_{\text {mean }}^{2} / \sigma^{2}$ is the error term optimized during BOBYQA, normalized with the noise level $\sigma . P(\boldsymbol{\theta})$ is the prior on the parameters of the model. We want to estimate the posterior distribution $P(\boldsymbol{\theta} \mid S)$. To do so, samples are drawn from the posterior probability using the Gaussian Process Hamiltonian Monte Carlo [42].

Hamiltonian Monte Carlo (HMC). HMC is a Markov Chain Monte Carlo algorithm which uses a refined proposal density function based on the Hamiltonian dynamics [43]. The idea is to have a high acceptance rate while proposing points relatively far from the current point. The problem is augmented with a momentum variable $\mathrm{p} \sim \mathcal{N}(\mathbf{0}, \mathbb{I})$. By randomly sampling $\mathbf{p}$, we define a current state $(\boldsymbol{\theta}, \mathbf{p})$. The energy of the state is $\mathrm{H}(\boldsymbol{\theta}, \mathbf{p})=\mathrm{E}_{\mathrm{pot}}+\mathrm{E}_{\mathrm{kin}}$, with potential energy $\mathrm{E}_{\mathrm{pot}}=-\log (P(S \mid \boldsymbol{\theta}) P(\boldsymbol{\theta}))$, and kinetic energy
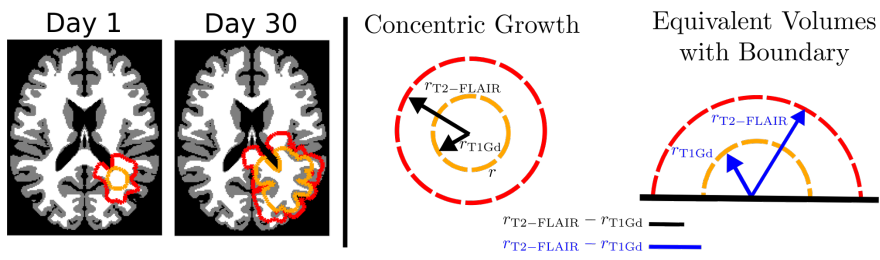

Figure 6: (Left) Synthetic growth of a tumor in the MNI atlas during 30 days with $D=1 \mathrm{~mm}^{2}$. day ${ }^{-1}$ and $\rho=0.18$ day $^{-1}$. The T2-FLAIR abnormality (isodensity of $\tau_{2}=16 \%$ ) is outlined in red. The T1Gd abnormality (isodensity of $\tau_{1}=80 \%$ ) is outlined in orange. (Right) Limits of the asymptotic personalization in presence of boundaries. The infiltration length - proportional to $r_{\mathrm{T} 2-\mathrm{FLAIR}}-r_{\mathrm{T} 1 \mathrm{Gd}}$ - tends to be under-estimated by considering the equivalent spherical volumes.

$\mathrm{E}_{\mathrm{kin}}=1 / 2\|\mathbf{p}\|_{2}^{2}$. Using the Hamiltonian dynamics for a certain period of time $\Delta t_{H}$,

$$
\frac{d \boldsymbol{\theta}_{i}}{d t}=\frac{\partial H}{\partial \mathbf{p}_{i}}, \quad \frac{d \mathbf{p}_{i}}{d t}=-\frac{\partial H}{\partial \boldsymbol{\theta}_{i}}
$$

a new state $\left(\boldsymbol{\theta}^{*}, \mathbf{p}^{*}\right)$ is proposed, with energy $\mathrm{H}\left(\boldsymbol{\theta}^{*}, \mathbf{p}^{*}\right)$. Using a Metropolis-Hastings acceptance criterion, the new state $\left(\boldsymbol{\theta}^{*}, \mathbf{p}^{*}\right)$ is accepted with probability $\mathcal{A}=$ $\min \left[1, \exp \left(-\mathrm{H}\left(\boldsymbol{\theta}^{*}, \mathbf{p}^{*}\right)+\mathrm{H}(\boldsymbol{\theta}, \mathbf{p})\right)\right]$. The conservation of the energy during the Hamiltonian dynamics - up to the numerical discretization accuracy of the Leapfrog scheme - insures a high acceptance rate $\mathcal{A}$, which is the ratio of proposed samples which are accepted. The boundary conditions on the bounded parameter space $\boldsymbol{\theta}$ are enforced using a bounce back condition [44] during the Leapfrog scheme used for the time integration. More precisely, if during the computations, the parameter $\boldsymbol{\theta}$ 


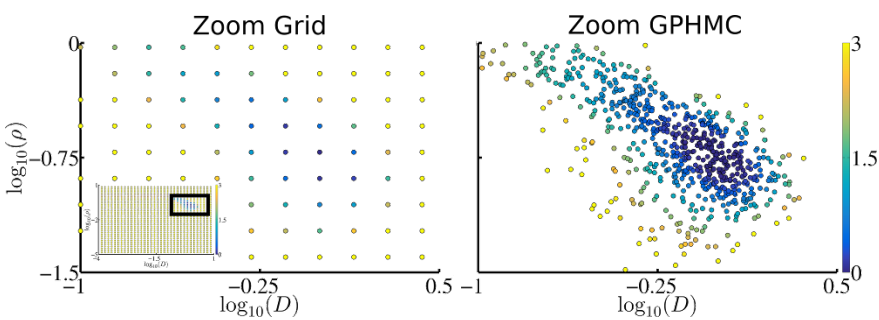

Figure 7: Comparison with a simple grid estimation of the posterior using $34 \times 34=1156$ model evaluations in the admissible domain for the synthetic case. The zoom to the region of low potential energy (i.e. high probability) proves the value of the presented method. The color scale indicates the negative log-likelihood.

crosses a boundary, its moment $\boldsymbol{p}$ is reversed and projected on the normal of the boundary.

Gaussian Process Hamiltonian Monte Carlo (GPHMC). In the HMC, computing the Hamiltonian dynamics - equations (9) - requires a significant amount of model evalutations. To circumvent this difficulty, $\mathrm{E}_{\mathrm{pot}}$ is approximated with a Gaussian process [42]. During the initialization phase, the forward model is evaluated on a coarse grid to initialize the Gaussian process. During the exploration phase, the forward model is evaluated at locations of low $\mathrm{E}_{\text {pot }}$ and high uncertainty on the Gaussian process interpolation (details can be found in [42]). $\mathrm{HMC}$ is then run using the Gaussian process interpolation of $\mathrm{E}_{\text {pot }}$ to compute the Hamiltonian dynamics. Given that the Gaussian process well captures $E_{\text {pot }}$, the GPHMC benefits from the high acceptance rate of the HMC, with far less model evaluations.

Parameters. The parameter $\boldsymbol{\theta}$ is constrained such that $D \in\left[10^{-4}, 10\right] \mathrm{mm}^{2} /$ days, and $\rho \in\left[10^{-5}, 10\right]$ days $^{-1}$, which encloses expected values for glioblastomas [21]. The prior $P(\boldsymbol{\theta})$ is assumed log-uniform within this bounded box. The Hamiltonian dynamics is run for $\Delta t_{H}=60$ days. For the likelihood, the noise level $\sigma$ has been empirically set to $5 \mathrm{~mm}$ to provide a reasonable acceptance rate. The noise level influences how peaked the estimated posterior will be. For the GPHMC, the Gaussian process is defined with a squared exponential covariance matrix $C\left(\boldsymbol{\theta}_{1}, \boldsymbol{\theta}_{2}\right)=$ $\left.w_{0} \exp \left(-\| \boldsymbol{\theta}_{1}-\boldsymbol{\theta}_{2}\right) \|_{2}^{2} / w_{1}^{2}\right)$ [45], where $\|.\|_{2}$ is the $l_{2}$ norm. These parameters are estimated with maximum likelihood [45]. During the GPHMC, the initialization is done with 49 forward model evaluations, while 50 evaluations are used for the exploratory phase, then 1000 samples are generated.

\section{RESULtS}

\section{A. Synthetic Case}

We perform a thorough analysis of a synthetic - but realistic - case. A simulation is run on the MNI atlas [46], with parameters $D=1 \mathrm{~mm}^{2}$.day ${ }^{-1}$ and $\rho=0.18$ day $^{-1}$, for 30 days (Figure 6). The result of the 3 different personalization methods can be seen on Figure 8. The result of the BOBYQA optimization is close to the mode of the computed posterior density. However, the spherical asymptotic analysis personalization is largely under-estimating the diffusion parameter $D$ with a solution of $D=0.41 \mathrm{~mm}^{2}$.day ${ }^{-1}$, when
$D=1.0$ and $0.96 \mathrm{~mm}^{2} \cdot$ day $^{-1}$ for the BOBYQA and the Maximum a Posteriori (MAP) sample of the Bayesian solution respectively. The asymptotic personalization method makes the assumption that the T1Gd and T2-FLAIR abnormalities are growing concentrically in an infinite domain. The presence of boundaries in realistic growth leads to the under-estimation of the difference of the radius of the abnormalities, leading to the under-estimation of the infiltration length (Figure 6).The acceptance rate during the GPHMC is $83 \%$, reflecting the fact that the Gaussian process is a good interpolation of the potential energy $\mathrm{E}_{\text {pot }}$ for the sampling.

The impact of the different parameters is analyzed on the synthetic case (Figure 8). We can see that using a uniform prior - like in [27] - results in samples which are more concentrated in the regions of high values of $D$ and $\rho$. However, it does not change much the shape and the location of the mode. On the other hand, using a noise level $\sigma=\sqrt{5} \mathrm{~mm}$ instead of $5 \mathrm{~mm}$ (with a log-uniform prior) results in a posterior which is much more peaked around the same mode. Finally, using the median Hausdorff distance instead of the 95th percentile (with a log-uniform prior and $\sigma=5 \mathrm{~mm}$ ) results in samples which are more spread because this distance is less discriminative. The effect is very similar to increasing the noise level $\sigma$. Finally, the method is compared to a simple grid evaluation of the potential energy for the synthetic case. The GPHMC automatically sample an extremely high density of points in the region of high probability, compared to the simplest grid approach (Figure 7).

\section{B. Glioblastoma Patients}

The method is applied to 7 patients. The T1Gd and T2FLAIR abnormalities were segmented by a clinician. A summary of the results can be found on Table I. The patients come from diverse clinical studies. We selected patients who were not treated with resection, and where two time points with visible growth were available. The patients were under chemotherapy, radiotherapy, and other specific types of therapy such as anti-angiogenic drug. This is one of the reasons why we focus on personalization, and not on prediction. The complex therapy schedule makes the prediction of the future behavior of the tumor difficult.

The result of the estimation can be seen on Figure 9 . The personalization based on the asymptotic analysis tends to provide low values of $D$ and $\rho$. We observe the variety of behavior of the posterior for the different patients. Figure 9 shows the best BOBYQA solution. The second and third best solutions are only shown if they are distinguishable with the first one at this scale, or if they fall in regions of low potential energy. The best BOBYQA solution always falls close to a mode of the posterior, but does not reflect the correlation between the parameters $D$ and $\rho$. Moreover, it is worth mentioning that for every patient, some of the differently initialized BOBYQA solutions lead to very poor results.

Patients 1, 2, and 3 present only one mode. The BOBYQA solution gives good results, while the asymptotic analysis always gives slightly off solutions. Patients 3,6 , and 7 present two modes. The two modes are very close to each other for 


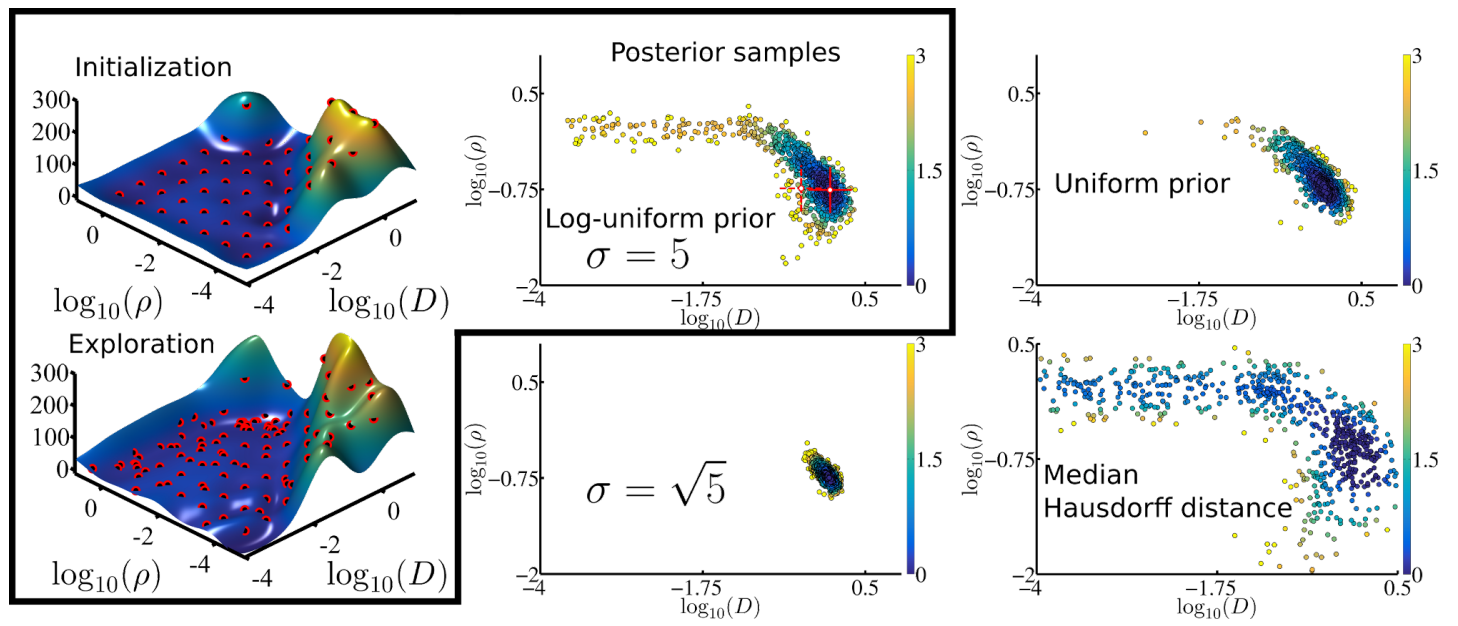

Figure 8: In the black box, (Left) i) Initialization of the Gaussian process interpolating the potential energy $\mathrm{E}_{\text {pot }}$ with a coarse $7 \times 7$ grid ii) Refinement of the Gaussian process with points of low potential energy and high uncertainty (see details in [42]) (Right) Personalization of the tumor growth model for the synthetic case. The result of the asymptotic personalization is at the intersection of the dashed red lines, the result of the BOBYQA optimization is at the intersection of the full red lines, and falls on the true parameters values at this scale. The color scale indicates the negative log-likelihood. Outside of the black box, results of the GPHMC personalization for the synthetic case using (from left to right, top to bottom) a uniform prior, a noise level $\sigma=\sqrt{5}$, and the median Hausdorff distance.

patients 6 and 7 while they are far apart for patient 3. This reflects the fact that the model cannot explain simultaneously the four available segmentations, and different sets of parameters lead to equivalently plausible solutions. For patient 5, the BOBYQA solutions and spherical asymptotic analysis give completely false result. This seems to be due to the fact that the solutions lie near the boundary of the domain, which lead to false convergence of the BOBYQA algorithm. For this patient, the parameters tend to lie at the edge of the admissible domain defined in Section IV-C.

The acceptance rate during the GPHMC is on average $72 \%$. This high acceptance rate is an indication that the Gaussian process is a good interpolation of the potential energy. However, for some patients, the interpolation is not as good resulting in acceptance rate of 52\% (Patient 3) and $56 \%$ (Patient 7). This is mainly due to narrow regions of high probability, where there are relatively few points to interpolate the potential energy. Note that the parameters of the GPHMC were the same for the seven different patients.

For a given patient, we can gather the samples in a matrix $\mathbf{X} \in \mathbb{R}^{n \times 2}$, where $n$ is the number of samples. We can project the samples in the $(\log (\lambda), \log (v))$ space, and compute the empirical covariance $C$,

$$
C=\operatorname{cov}\left(X\left(\begin{array}{cc}
\frac{1}{2} & \frac{1}{2} \\
-\frac{1}{2} & \frac{1}{2}
\end{array}\right)\right)
$$

We then compute the normalized variances $\sigma_{\text {inv }}$ and $\sigma_{\text {speed }}$ of the posterior samples along the speed and invisibility index axis respectively,

$$
\sigma_{\text {inv }}=\frac{C(1,1)}{\operatorname{Tr}[C]}, \quad \sigma_{\text {speed }}=\frac{C(2,2)}{\operatorname{Tr}[C]}
$$

where $\operatorname{Tr}[$.$] refers to the trace of the matrix. This way,$ we can quantify which of the invisibility index or speed is better captured by the model. For instance, patient 3 presents

\begin{tabular}{|l|c|c|c|c|}
\hline & $t_{2}-t_{1}$ (days) & Acceptance rate (\%) & $\sigma_{\text {speed }}(\%)$ & $\sigma_{\text {inv }}(\%)$ \\
\hline Synthetic & 30 & 83 & 23 & 77 \\
\hline Pat. 1 & 105 & 84 & 45 & 55 \\
\hline Pat. 2 & 29 & 87 & 74 & 26 \\
\hline Pat. 3 & 26 & 52 & 99 & 1 \\
\hline Pat. 4 & 31 & 72 & 98 & 2 \\
\hline Pat. 5 & 14 & 77 & 98 & 2 \\
\hline Pat. 6 & 28 & 74 & 82 & 18 \\
\hline Pat. 7 & 29 & 56 & 83 & 17 \\
\hline
\end{tabular}

Table I: Results on 7 patients: time span between the time points; acceptance rate of the GPHMC; percentage of variance due to the speed; percentage of variance due to the invisibility index.

an extremely elongated posterior along the line of constant invisibility index $\left(\sigma_{\mathrm{inv}}=1 \%, \sigma_{\text {speed }}=99 \%\right)$, showing that the uncertainty on the fit is due to the speed rather than the invisibility index. For patient 1 , the posterior is not as elongated, showing that the uncertainty due to the speed or the invisibility index are more similar $\left(\sigma_{\mathrm{inv}}=45 \%\right.$, $\sigma_{\text {speed }}=55 \%$ ). This is probably caused by the fact that the two time points are 105 days apart for patient 1 . As such the speed of growth of the tumor is more identifiable resulting in a drop of the variance due to the speed compared to the other patients. Similarly, the synthetic case presents a larger growth which makes the identifiability of the speed easier.

We show the results of the simulation using the parameter $\boldsymbol{\theta}$ corresponding to the Maximum a Posteriori (MAP) of the samples (Figure 10) for two representative patients. Furthermore, for each sample resulting from the GPHMC, one can compute the corresponding segmentation for the $\mathrm{T} 1 \mathrm{Gd}$ at the second time point, and the T2-FLAIR at the first and second time points. We can then compute the probability for each voxel to lie within one of those segmentations. We show on Figure 11 the 10\% (in blue) and 90\% (in green) isolines of this probability mask which allows to visualize the uncertainty in the MAP segmentation. 



Figure 9: Personalization of the tumor growth model for the 7 patients. The result of the asymptotic personalization is at the intersection of the dashed red lines, the result of the BOBYQA optimization is at the intersection of the solid red lines. The presence of several BOBYQA solutions from different initializations is indicated when the best three solutions were noticeably distinct. The color scale indicates the negative log-likelihood of the posterior $P(\boldsymbol{\theta} \mid S)$, which is equal to the potential energy $\mathrm{E}_{\text {pot }}$. 


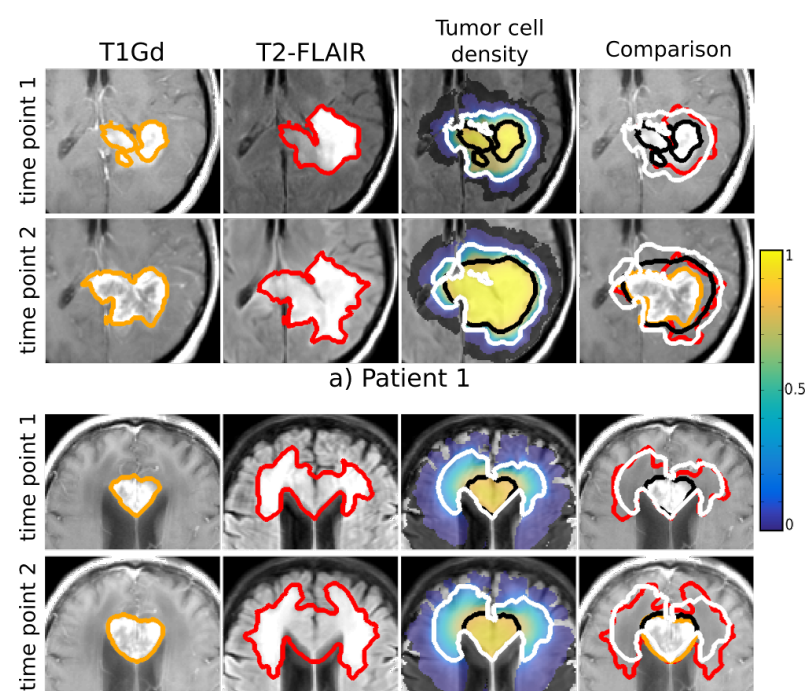

b) Patient 4

Figure 10: Maximum a Posteriori fit for Patient 1 (Top) and Patient 4 (Bottom). For each patient: (Left) The proliferative rim is outlined in orange on the T1Gd MRI at two different time points; (Middle Left) The edema is outlined in red on the T2-FLAIR MRI at two different time points; (Middle Right) Tumor cell density, the black (resp. white) line is the threshold value $\tau_{1}$ (resp. $\tau_{2}$ ) corresponding to the T1Gd (resp. T2-FLAIR) abnormality; (Right) Comparison between the clinician segmentation and the contours from the model.

\section{DISCUSSION}

In this work, we presented an efficient implementation of the reaction-diffusion equation for the brain tumor growth based on the Lattice Boltzmann Method. We further presented estimation methods of the model's parameters of different levels of complexity. The simplest one is based on the asymptotic properties of the reaction-diffusion equation. It does not require complex computations, but fails when the growth is constrained by the brain boundaries. The second method is the optimization of an error term using a derivative-free algorithm. In our experiments, the method required on average 20 model evaluations per initialization, resulting in 180 model evaluation for a total of 9 different initializations. The third method is based on an efficient Monte Carlo method called the Gaussian Process Hamiltonian Monte Carlo, used to sample from a posterior, derived as the Boltzmann distribution of the previous error term. It requires a total of 1100 model evaluations. As such, the estimation of the posterior probability requires only 6 times more evaluations than the direct optimization, and provides additional valuable informations about the shape of the posterior. This reveals the possible presence of several modes, and the correlation between the parameters due to the lack of identifiability of the speed or the invisibility index.

Moreover, the samples of the posterior density could be used to estimate the density in the whole domain using for instance kernel density estimation [27]. This could be helpful to have access to other useful statistical indices such as the evidence of the model, which can help answer questions about model selection [47], [27].

In the future, we could consider the thresholds $\tau_{1}$ and $\tau_{2}$ as parameters of the model to be sampled. This could also be

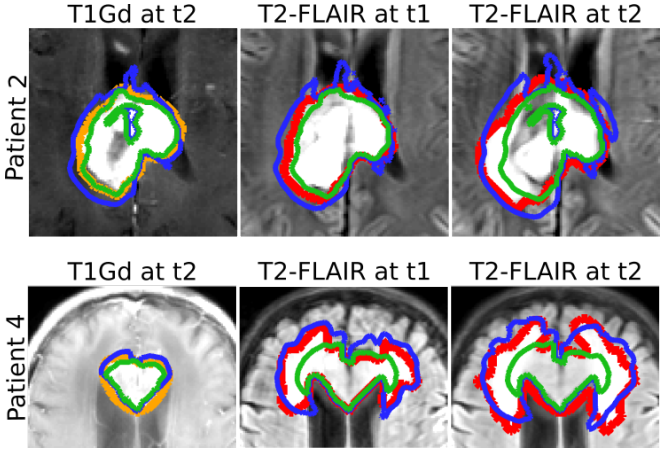

Figure 11: (Top) Patient 2 (Bottom) Patient 4. From left to right: T1Gd for the second time point, T2-FLAIR for the first time point, T2-FLAIR for the second time point. The clinician segmentation are in orange for the T1Gd and red for the T2-FLAIR.The blue outline (resp. green) encloses the voxels which were present in at least $10 \%$ (resp. 90\%) of the segmentations deduced from the samples.

the case for the noise level $\sigma$. Moreover, the modeling of the mass effect could be useful in order to avoid the non-linear registration between the second and the first time points.

\section{Perspectives}

In the future, we intend to apply the Bayesian personalization in order to explicitly take into account the uncertainty in the expert's segmentation. More specifically, the segmentations used during each model evaluation could be sampled in the space of plausible segmentations [48]. We also believe that this work could be used for automatic personalized therapy planning. Some work has already been done on relating tumor growth models to radiation response models to better define radiation therapy plans [49], [50], [6]. Such a method could provide personalized therapy plans taking into account the uncertainty in the model's parameters.

\section{ACKNOWLEDGMENT}

Part of this work was funded by the European Research Council through the ERC Advanced Grant MedYMA 2011-291080.

\section{REFERENCES}

[1] F. Raman, E. Scribner, O. Saut, C. Wenger, T. Colin, and H. M. Fathallah-Shaykh, "Computational trials: Unraveling motility phenotypes, progression patterns, and treatment options for glioblastoma multiforme," PloS one, vol. 11, no. 1, 2016.

[2] M. L. Neal, A. D. Trister, S. Ahn, A. Baldock, C. A. Bridge, L. Guyman, J. Lange, R. Sodt, T. Cloke, A. Lai, T. F. Cloughesy et al., "Response classification based on a minimal model of glioblastoma growth is prognostic for clinical outcomes and distinguishes progression from pseudoprogression," Cancer research, vol. 73, no. 10, pp. 2976-2986, 2013.

[3] C. H. Wang, J. K. Rockhill, M. Mrugala, D. L. Peacock, A. Lai, K. Jusenius, J. M. Wardlaw, T. Cloughesy, A. M. Spence et al., "Prognostic significance of growth kinetics in newly diagnosed GBM revealed by combining serial imaging with a novel biomathematical model," Cancer research, vol. 69, no. 23, pp. 9133-9140, 2009.

[4] E. Stretton, E. Mandonnet, E. Geremia, B. H. Menze, H. Delingette, and N. Ayache, "Predicting the location of glioma recurrence after a resection surgery," in Spatio-temporal Image Analysis for Longitudinal and Time-Series Image Data. Springer, 2012, pp. 113-123.

[5] D. Corwin, C. Holdsworth, R. C. Rockne, A. D. Trister, M. M. Mrugala, J. K. Rockhill, R. D. Stewart, M. Phillips, and K. R. Swanson, "Toward patient-specific, biologically optimized radiation therapy plans for the treatment of glioblastoma," PloS one, vol. 8, no. 11, p. e79115, 2013. 
[6] R. Rockne, E. Alvord Jr, J. Rockhill, and K. Swanson, "A mathematical model for brain tumor response to radiation therapy," Journal of mathematical biology, vol. 58, no. 4-5, pp. 561-578, 2009.

[7] T. A. Dolecek, J. M. Propp, N. E. Stroup, and C. Kruchko, "CBTRUS statistical report: primary brain and central nervous system tumors diagnosed in the United States in 2005-2009," Neuro-oncology, vol. 14, pp. v1-v49, 2012.

[8] E. D. Angelini, O. Clatz, E. Mandonnet, E. Konukoglu, L. Capelle, and H. Duffau, "Glioma dynamics and computational models: a review of segmentation, registration, and in silico growth algorithms and their clinical applications," Current Medical Imaging Reviews, vol. 3, no. 4, pp. 262-276, 2007.

[9] J. Murray, Mathematical biology. Springer, 2002, vol. 2.

[10] P. Tracqui, G. Cruywagen, D. Woodward, G. Bartoo, J. Murray, and E. Alvord, "A mathematical model of glioma growth: the effect of chemotherapy on spatio-temporal growth," Cell proliferation, vol. 28 , no. 1, pp. 17-31, 1995.

[11] K. R. Swanson, E. Alvord, and J. Murray, "Virtual brain tumours (gliomas) enhance the reality of medical imaging and highlight inadequacies of current therapy," British journal of cancer, vol. 86, no. 1, pp. 14-18, 2002.

[12] M. Chaplain, "Avascular growth, angiogenesis and vascular growth in solid tumours: The mathematical modelling of the stages of tumour development," Mathematical and computer modelling, vol. 23, no. 6, pp. 47-87, 1996.

[13] E. Konukoglu, O. Clatz, B. H. Menze, B. Stieltjes, M.-A. Weber, E. Mandonnet, H. Delingette, and N. Ayache, "Image guided personalization of reaction-diffusion type tumor growth models using modified anisotropic eikonal equations," IEEE TMI, vol. 29, no. 1, pp. 77-95, 2010.

[14] P. Mosayebi, D. Cobzas, A. Murtha, and M. Jagersand, "Tumor invasion margin on the riemannian space of brain fibers," Medical image analysis, vol. 16, no. 2, pp. 361-373, 2012.

[15] O. Clatz, M. Sermesant, P.-Y. Bondiau, H. Delingette, S. K. Warfield, G. Malandain, and N. Ayache, "Realistic simulation of the 3-D growth of brain tumors in MR images coupling diffusion with biomechanical deformation," IEEE TMI, vol. 24, no. 10, pp. 1334-1346, 2005.

[16] C. Hogea, C. Davatzikos, and G. Biros, "An image-driven parameter estimation problem for a reaction-diffusion glioma growth model with mass effects," Journal of mathematical biology, vol. 56, no. 6, pp. 793825,2008

[17] K. Swanson, R. Rockne, J. Claridge, M. Chaplain, E. Alvord Jr, and A. Anderson, "Quantifying the role of angiogenesis in malignant progression of gliomas: In silico modeling integrates imaging and histology," Cancer research, vol. 71, no. 24, pp. 7366-7375, 2011.

[18] O. Saut, J.-B. Lagaert, T. Colin, and H. M. Fathallah-Shaykh, "A multilayer grow-or-go model for gbm: effects of invasive cells and anti-angiogenesis on growth," Bulletin of mathematical biology, vol. 76, no. 9, pp. 2306-2333, 2014.

[19] A. Hawkins-Daarud, R. C. Rockne, A. R. Anderson, and K. R. Swanson, "Modeling tumor-associated edema in gliomas during anti-angiogenic therapy and its impact on imageable tumor," Frontiers in oncology, vol. 3, 2013 .

[20] M. Badoual, C. Gerin, C. Deroulers, B. Grammaticos, J.-F. Llitjos, C. Oppenheim, P. Varlet, and J. Pallud, "Oedema-based model for diffuse low-grade gliomas: application to clinical cases under radiotherapy," Cell proliferation, vol. 47, no. 4, pp. 369-380, 2014.

[21] H. L. Harpold, E. C. Alvord Jr, and K. R. Swanson, "The evolution of mathematical modeling of glioma proliferation and invasion," Journal of Neuropathology \& Experimental Neurology, vol. 66, no. 1, 2007.

[22] M. J. Powell, "The BOBYQA algorithm for bound constrained optimization without derivatives," Cambridge NA Report NA2009/06, 2009.

[23] A. Gooya, K. M. Pohl, M. Bilello, L. Cirillo, G. Biros, E. R. Melhem, and C. Davatzikos, "GLISTR: glioma image segmentation and registration," IEEE TMI, vol. 31, no. 10, pp. 1941-1954, 2012.

[24] B. H. Menze, K. Van Leemput, A. Honkela, E. Konukoglu, M.-A. Weber, N. Ayache, and P. Golland, "A generative approach for image-based modeling of tumor growth," in IPMI. Springer, 2011, pp. 735-747.

[25] E. Konukoglu, J. Relan, U. Cilingir, B. H. Menze, P. Chinchapatnam, A. Jadidi, H. Cochet, M. Hocini, H. Delingette, P. Jaïs et al., "Efficient probabilistic model personalization integrating uncertainty on data and parameters: Application to eikonal-diffusion models in cardiac electrophysiology," Progress in biophysics and molecular biology, vol. 107, no. 1, pp. 134-146, 2011.

[26] D. Neumann, T. Mansi, B. Georgescu, A. Kamen, E. Kayvanpour A. Amr, F. Sedaghat-Hamedani, J. Haas, H. Katus, B. Meder et al., "Robust image-based estimation of cardiac tissue parameters and their uncertainty from noisy data," in MICCAI. Springer, 2014, pp. 9-16.
[27] M. Lê, H. Delingette, J. Kalpathy-Cramer, E. Gerstner, T. Batchelor, J. Unkelbach, and N. Ayache, "Bayesian personalization of brain tumor growth model," in MICCAI. Springer, 2015.

[28] E. Stretton, E. Geremia, B. H. Menze, H. Delingette, and N. Ayache, "Importance of patient DTI's to accurately model glioma growth using the reaction diffusion equation," in IEEE ISBI. San Francisco, CA, United States: IEEE, 2013, pp. 1130-32.

[29] F. Dittmann, B. H. Menze, E. Konukoglu, and J. Unkelbach, "Use of diffusion tensor images in glioma growth modeling for radiotherapy target delineation," in Multimodal Brain Image Analysis. Springer, 2013, pp. 63-73.

[30] M. Jenkinson, P. Bannister, M. Brady, and S. Smith, "Improved optimization for the robust and accurate linear registration and motion correction of brain images," Neuroimage, vol. 17, no. 2, 2002.

[31] J. L. Andersson, M. Jenkinson, S. Smith et al., "Non-linear registration, aka spatial normalisation fmrib technical report tr07ja2," FMRIB Analysis Group of the University of Oxford, 2007.

[32] M. Jenkinson, C. F. Beckmann, T. E. Behrens, M. W. Woolrich, and S. M. Smith, "FSL," Neuroimage, vol. 62, no. 2, pp. 782-790, 2012.

[33] J. E. Iglesias, C.-Y. Liu, P. M. Thompson, and Z. Tu, "Robust brain extraction across datasets and comparison with publicly available methods," IEEE TMI, vol. 30, no. 9, pp. 1617-1634, 2011.

[34] Y. Zhang, M. Brady, and S. Smith, "Segmentation of brain mr images through a hidden markov random field model and the expectationmaximization algorithm," IEEE TMI, vol. 20, no. 1, pp. 45-57, 2001.

[35] L. Zhao, U. Ruotsalainen, J. Hirvonen, J. Hietala, and J. Tohka, "Automatic cerebral and cerebellar hemisphere segmentation in 3D MRI: adaptive disconnection algorithm," Medical image analysis, vol. 14, no. 3, pp. 360-372, 2010.

[36] E. Özuğurlu, "A note on the numerical approach for the reactiondiffusion problem to model the density of the tumor growth dynamics," Computers \& Mathematics with Applications, 2015.

[37] S. Rapaka, T. Mansi, B. Georgescu, M. Pop, G. A. Wright, A. Kamen, and D. Comaniciu, "LBM-EP: lattice-Boltzmann method for fast cardiac electrophysiology simulation from 3D images," in MICCAI 2012. Springer, 2012, pp. 33-40.

[38] C. Audigier, T. Mansi, H. Delingette, S. Rapaka, V. Mihalef, D. Carnegie, E. Boctor, M. Choti, A. Kamen, N. Ayache et al., "Efficient lattice Boltzmann solver for patient-specific radiofrequency ablation of hepatic tumors," IEEE TMI, p. 14, 2015.

[39] H. Yoshida and M. Nagaoka, "Multiple-Relaxation-Time LBM for the convection and anisotropic diffusion equation," Journal of Computational Physics, vol. 229, no. 20, 2010

[40] D. Yu, R. Mei, L.-S. Luo, and W. Shyy, "Viscous flow computations with the method of lattice Boltzmann equation," Progress in Aerospace Sciences, vol. 39, no. 5, pp. 329-367, 2003.

[41] E. Konukoglu, O. Clatz, P.-Y. Bondiau, H. Delingette, and N. Ayache, "Extrapolating glioma invasion margin in brain magnetic resonance images: Suggesting new irradiation margins," Medical image analysis, vol. 14, no. 2, 2010.

[42] C. E. Rasmussen, "Gaussian processes to speed up hybrid Monte Carlo for expensive Bayesian integrals," vol. 7, 2003, pp. 651-659.

[43] R. M. Neal, "MCMC using Hamiltonian dynamics," Handbook of Markov Chain Monte Carlo, vol. 2, 2011.

[44] M. Betancourt, "Nested sampling with constrained hamiltonian monte carlo," arXiv preprint arXiv:1005.0157, 2010.

[45] C. E. Rasmussen, "Gaussian processes for machine learning," 2006.

[46] J. Mazziotta, A. Toga, A. Evans, P. Fox, J. Lancaster, K. Zilles, R. Woods, T. Paus, G. Simpson, B. Pike et al., "A probabilistic atlas and reference system for the human brain: International consortium for brain mapping (icbm)," Philosophical Transactions of the Royal Society B: Biological Sciences, vol. 356, no. 1412, pp. 1293-1322, 2001.

[47] J. T. Oden, E. E. Prudencio, and A. Hawkins-Daarud, "Selection and assessment of phenomenological models of tumor growth," Mathematical Models and Methods in Applied Sciences, vol. 23, no. 07, 2013.

[48] M. Lê, J. Unkelbach, N. Ayache, and H. Delingette, "GPSSI: Gaussian process for sampling segmentations of images," in MICCAI. Springer, 2015.

[49] J. Unkelbach, B. H. Menze, E. Konukoglu, F. Dittmann, M. Le, N. Ayache, and H. A. Shih, "Radiotherapy planning for glioblastoma based on a tumor growth model: improving target volume delineation," Physics in medicine and biology, vol. 59, no. 3, p. 747, 2014.

[50] J. Unkelbach, B. H. Menze, E. Konukoglu, F. Dittmann, N. Ayache, and H. A. Shih, "Radiotherapy planning for glioblastoma based on a tumor growth model: implications for spatial dose redistribution," Physics in medicine and biology, vol. 59, no. 3, p. 771, 2014. 